\section{CONNECT WITH OTHER BDA MEMBERS ON THE GO}

The British Dental

Association (BDA) has

launched a new social media platform for members called BDA Connect. The platform hosts blogs from leading members of the dental community and forums for the exchange of news and views.

BDA Connect is built on 'responsive design' and will function as well on smaller-screened

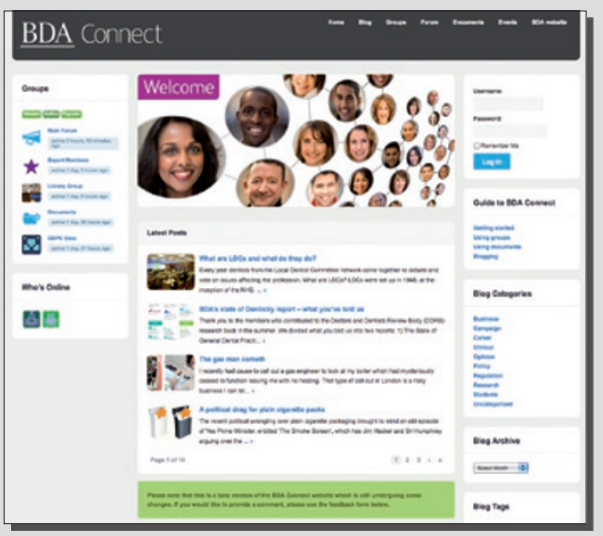
mobile and tablet devices as on laptop and desktop computers, offering convenience and accessibility to users. Its functionality also allows members to choose how often and on what topics they receive updates on activity on the site.

Dr Martin Fallowfield, the Chair of the BDA's Principal Executive Committee, said: 'Connect is a new place where the profession can meet, debate and share news, views and experiences. In creating it we have completely overhauled our previous communities site to provide a mobile, adaptable and user-friendly platform that will better meet the needs of our members. We look forward to welcoming members to Connect'.

BDA Connect can be accessed via the BDA website at: https://bdaconnect.bda.org/.

\section{DENTISTS ADDRESS THE HAGGIS}

The BDA Northern Counties Branch held its Burns Night event at The Assembly Rooms in Newcastle on Saturday 25 January 2014. The event was much larger this year after the inaugural event last year, with over 170 people in attendance this time. Professor Jimmy Steele CBE, Dean of Newcastle Dental School, delivered the Address to the Haggis in his own theatrical style.

The event was hosted by Paul Blaylock, the Northern Counties Branch President. Speakers also included Dr John Meechan, Clinical Lead for Oral and Maxillofacial Surgery at Newcastle Dental School, who gave the Immortal Memory to Robbie Burns. This included a rendition of his own poem in the style of Burns, celebrating the Scottish credentials of Mel Gibson following his appearance in Braveheart.

In addition to the speeches, all the guests enjoyed a taste of haggis as part of the traditional Burns Night meal. The event was rounded off with a ceilidh late into the evening. The Branch looks forward to hosting a similar event in future.

\section{FIRST EVER SYMPOSIUM} ON ORAL HEALTH IN SPORT

On 4 April 2014, UCL Eastman Dental Institute and the UCL Institute of Sport, Exercise \& Health will host the first ever symposium on oral health and elite sport performance to be held in the UK.

Oral Health and Performance in Sport - Learning from London 2012 will take place in central London and will feature presentations from a wide range of speakers, including sports physicians and scientists, Olympic team dentists and doctors, leading oral health researchers, and representatives from the English Institute of Sport.

The event is designed to appeal to anyone with an interest in the relationship between oral health and elite performance, and the organisers hope it will foster new collaborations and new thinking in this field.

Register before 28 February 2014 for discounted entry at: www. ucl.ac.uk/eastman/cpd/events/oralhealth-and-performance-in-sport.

\section{BOOK REVIEW}

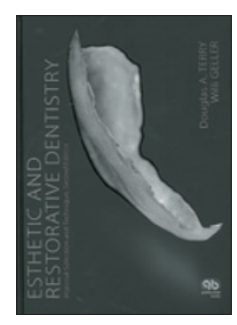

ESTHETIC AND RESTORATIVE DENTISTRY: MATERIAL SELECTION AND TECHNIQUE, 2ND EDITION
D. A. Terry, W. Geller (eds)
Quintessence
price $€ 220.00$; pp 752
ISBN 9780867155730

This book aims to help the clinician meet the increasing high expectation of patients for aesthetic results by enhancing the graduate's ability to provide high-quality and comprehensive restorative management for most aesthetic cases. Though over 700 pages, it is well laid out with vibrant step-bystep high quality clinical photographs accompanying the detailed evidence based content. This brings the clinical information to life and engages the reader. It also illustrates an impeccable standard of aesthetic cases. The target audience would be postgraduates looking to enhance their restorative cosmetic skills.

The author provides handy tips on shade analysis, lab communication and instrument position for creating optimal aesthetic results. From simplified to more advanced layering techniques the entire protocol for composite restorations from Class I through IV including direct veneers are vividly demonstrated. Answers to restorative challenges related to impression taking are given. There's a guide for selecting optimum luting agents for different clinical scenarios. A simplified table of design principles for the adhesive post-retained system is provided, with descriptive illustrations of the ferrule effect.

The author explains the interplay between bio-modification of tooth tissue and adhesion mechanisms. Both total etch and self-etch protocol is clearly illustrated for direct composite restorations and the cementation of indirect restorations so a durable adhesion between the tooth and material is achieved. The various types of hand and rotary instruments for finishing and polishing are illustrated photographically. The author describes the exact angle of instrumentation.

A great passion of mine is dental photography, which other restorative books lack detail on. The author dedicates a chapter to this and has enhanced my own knowledge. Periodontal surgical methods such as clinical crown lengthening, sub-epithelial connective tissues grafting and hard and soft tissue ridge augmentation are explained well. The current implant bio-integration concepts are described in detail. The final chapter on bio-modification of tooth discoloration discusses bleaching technique. However, this is an American text so the UK dentist should be aware of the different legislation on maximum peroxide concentration when bleaching.

I enjoyed this book. The authors' wealth of knowledge is impressive. I have no hesitation in recommending it; it is a must on the bookshelf of any aesthetic restorative dentist.

B. BAJOMO 